\title{
Collaborative pharmacy practice: an update
}

This article was published in the following Dove Press journal:

Integrated Pharmacy Research and Practice

22 June 2013

Number of times this article has been viewed

\section{Anandi V Law \\ Eric K Gupta \\ Micah Hata \\ Karl M Hess \\ Roger S Klotz \\ Quang A Le \\ Emmanuelle Schwartzman \\ Bik-Wai Bilvick Tai}

Department of Pharmacy Practice and Administration, College of Pharmacy, Western University of Health Sciences, Pomona, CA, USA
Correspondence: Anandi V Law

Department of Pharmacy Practice and Administration, College of Pharmacy,

Western University of Health Sciences,

309 E Second Street, Pomona,

CA 91766 , USA

Tel +l 9094695645

Fax + I 9094695428

Email alaw@westernu.edu
Abstract: Collaborative practice among health professionals is slowly coming of age, given the global focus on efficiency and effectiveness of care to achieve positive patient outcomes and to reduce the economic burden of fragmented care. Collaborative pharmacy practice (CPP) is accordingly evolving within different models including: disease management, medication therapy management, patient centered medical home, and accountable care organizations. Pharmacist roles in these models relate to drug therapy management and include therapy introduction, adjustment, or discontinuation, patient counseling and education, and identification, resolution, and prevention of problems leading to drug interactions and adverse reactions. Most forms of CPP occur with physicians in various settings. Collaborative practice agreements exist in many states in the US and are mentioned in the International Pharmaceutical Federation policy statement. Impetus for CPP comes from health system and economic concerns, as well as from a regulatory push. There are positive examples in community, ambulatory care, and inpatient settings that have well documented protocols, indicators of care, and measurement and reporting of clinical, economic, and patient reported outcomes; however, implementation of the practice is still not widespread. Conceptual and implementation challenges include health professional training, attitudes, confidence and comfort levels, power and communication issues, logistic barriers of time, workload, proximity, resistance to establish and adopt regulations, and importantly, payment models. Some of the attitudinal and perceptual challenges can be mitigated by incorporation of interprofessional concepts and practice in health profession education. Other challenges need to be addressed across health systems, given the inefficiencies and problems that arise from lack of communication and coordination of patient care including medication nonadherence, errors and patient safety, complexity of compounded health problems, and potential liability. The existing evidence needs to be examined to address some challenges and improve infrastructure for CPP.

Keywords: collaborative pharmacy practice, collaboration model, interprofessional education, collaborative patient care, coordination of care, continuity of care

\section{Background}

In his book on sociological change, Malcolm Gladwell defines a tipping point as "the moment of critical mass, the threshold, the boiling point," when ideas or messages spread to reach the point where a movement or change occurs. ${ }^{1}$ A similar pattern can be drawn for collaborative patient care in the US. As health care costs increased exponentially through the turn of the twentieth century, and several factors including an aging population, chronic conditions, and the cost of delivery of complex care projected an upward trend in future costs, the system began to review solutions. Adding to the issue was the burgeoning nonadherence to therapy, error rate in health 
care delivery, and related suboptimal health outcomes. ${ }^{2}$ In March 2001, a report titled "Crossing the quality chasm: a new health system for the 21 st century" by the Institute of Medicine, which is an entity of multidisciplinary experts, identified a gap (chasm) in the US health care system that prevents patients from receiving the care that the system is capable of providing. ${ }^{3}$ In describing the multiple levels of systemic change necessary to achieve an improved quality of care (safe, effective, patient centered, timely, efficient, and equitable care) for patients with chronic diseases, the report presented specific areas for systemic redesign including information technology, alignment of payment with quality improvement, and more importantly, training of health professionals in interdisciplinary and evidence based practice. This report, which appears to be the tipping point in the awareness for collaborative patient care, shed light on the need for communication and collaboration among health professionals as a means of establishing much needed continuity and coordination of care to achieve positive patient care outcomes.

\section{Objectives}

This review aimed to present an update on collaborative pharmacy practice (CPP); accordingly, the outline of the paper includes definition, description and evolution, theories and models of collaborative practice, best practices, challenges in its implementation, and future directions in the area.

\section{Introduction}

Collaborative patient care practice is focused on a team based approach to health care of a patient and their family to ultimately achieve a higher level of continual care. ${ }^{3}$ Its genesis in the US goes back to World War II when different medical professionals worked together as a team to provide effective treatment to wounded soldiers. ${ }^{4}$ However, adoption of the model in concept and implementation has been delayed due to the absence of pertinent state laws and regulations, the era of super specialization in each health profession, resistance by providers who felt threatened, and the lack of a compensation mechanism from third parties for such care. Recent impetus for collaborative practice has been from regulatory and national quality organizations. As evidence began to highlight the correlation between the absence of continuous, coordinated, and collaborative care with negative outcomes, collaborative practice within and among different health professionals has now become a national agenda. The newly emerging team based approach brings health professionals, like physicians, pharmacists, and nurses, together by engaging them in the provision of care. As such, in interdisciplinary collaborative teams, every health care professional has access to important patient data (ie, laboratory data, total medication history, physical assessment data) and is therefore aware of a patient's complete health care needs and can reasonably expect the other providers' actions and procedures within the framework of collaborative health care. ${ }^{5}$

\section{Search strategy for review}

A structured search was performed focusing on the characteristics of CPP. English language articles were identified in Pubmed up to October 2012 using the following search terms: "collaborative pharmacy practice", "interdisciplinary pharmacy practice", "multidisciplinary pharmacy practice", "physician pharmacist collaboration" "collaborative drug therapy management", "collaborative practice agreement", "physician pharmacist agreement", "physician pharmacist partnership", "physician pharmacist model", "physician pharmacist outcome", "pharmacy collaborative care example", "collaborative practice reimbursement", and "medication therapy management". The references of identified articles were also reviewed to identify other potentially relevant publications.

Letters, notes, and conference abstracts were excluded. In total, 142 full text articles were retrieved for review, and 55 were included for reference in the manuscript preparation. Additional sources such as books, electronic news, organization policy statements, reports, and websites relevant to the topic were also included for reference.

\section{Description of CPP}

CPP has been variable for several reasons. The often noted cliché in health care has been the presence of silos in practice. Pharmacists typically focused on prescription dispensing secondary to physician diagnosis and prescribing. Collaborative practice between pharmacists and physicians may have been present episodically or anecdotally, but was not actively pursued until patient safety and outcomes became the focus of health care. The medication focused nature of the pharmacy profession has led to definitions and examples of CPP that are centered on physician-pharmacist collaboration.

Collaborative practice involving pharmacists has been gradually gaining traction based on evidence and regulatory forces. The Collaborative Practice Agreement Act (CPAAct) that currently exists in 46 US states gives pharmacists permission to voluntarily enter into collaborative agreements 
with physicians and other providers to offer a variety of health care services to patients according to a set protocol. ${ }^{6,7}$ In most US states that allow collaborative management, no special requirements beyond licensure are required to enter into such an arrangement. However, in a few US states, completion of board certification, an American Society of Health-System Pharmacists (ASHP) accredited residency, a certain number of years working as a licensed pharmacist (Bachelor of Pharmacy or Doctor of Pharmacy), and/or working in certain settings such as in a clinic or in long-term care are required. ${ }^{8}$

Collaborative practice between a pharmacist and a physician focuses on drug therapy management which includes medication therapy introduction, adjustment, or discontinuation, patient counseling/education, and lastly, the identification, resolution, and prevention of any drug-drug interactions, as well as the identification of adverse reactions via symptom analysis by the pharmacist, thus eliminating a potential diagnostic problem. Most forms of collaborative practice appear to involve pharmacists working with a single physician or groups of physicians in a medical office, clinic, or other ambulatory care type setting. ${ }^{9-12}$ Similar to the CPAAct, pharmacists in other countries have agreements with physicians; for example, in Quebec, Canada, pharmacists are allowed to initiate and modify drug therapy based on a physician's prescription and request laboratory analyses as needed. $^{13}$

\section{Process models for CPP}

This section presents an overview of the different types of collaborative processes that exist in practice or that are emerging. Disease states for which collaborative practice models have been reported include dyslipidemia, hypertension, metabolic syndrome, and chronic kidney disease. ${ }^{11,13-16}$

In these reports, ${ }^{11,13-16}$ the overall model is such that the patients' first access to healthcare is through their physician. The physician, in turn, delegates certain responsibilities to the pharmacist, depending upon the exact collaborative practice arrangement which has been made between the physician and pharmacist, in an effort to attain better clinical outcomes for their patients. Pharmacist responsibilities within these reports range from identifying and resolving singular medication therapy problems and prescribing medication renewals to tasks such as adding, deleting, or otherwise adjusting the medication therapy regimens of patients, ordering laboratory tests, and performing limited physical assessments. Through the incorporation of pharmacists, medication management is typically better optimized and patient care outcomes improved. Typically, since physicians and pharmacists work in close proximity in these types of settings, interprofessional communication is improved, thus further fostering collaboration and improving patient care. ${ }^{17}$

CPP tends to be a global description encompassing many forms of collaborative practice. Some of the delivery models of care that exist or are emerging and that involve pharmacists include: disease management (DM), medication therapy management (MTM), patient centered medical home (PCMH), accountable care organizations (ACOs). Each of these models has some characteristics that set it apart but they all revolve around the concept of collaborative practice to ensure optimal outcomes. Delivery of care in each model includes providerprovider and provider-patient communication, coordination, and continuity of care. In DM, the focus is on patients with a specific highly prevalent chronic condition that is highly associated with multiple and complex morbidities if untreated or uncontrolled, has multiple modalities of treatment and care, has potential for self-care, and has a significant economic burden. ${ }^{18,19}$ Pharmacists have been shown to improve management of the condition and resultant clinical and patient reported outcomes, along with reduced economic burden when they provide patient education on medications, continuous monitoring in conjunction with physicians, and close the loop on communication - front- and back-end. Further, streamlined protocols of care, specified indicators of change, and documentation of outcomes have been essential aspects of successful DM programs. ${ }^{20,21}$

MTM is another model that was officially established as a program by the Center for Medicare and Medicaid Services (CMS) as part of the Medicare Modernization and Improvement Act (2003) to improve medication adherence and optimize therapeutic outcomes. ${ }^{22}$ In particular, MTM has provided an opportunity for pharmacists to provide more direct patient care services in a community setting. According to the American Pharmacists Association, MTM services may include: medication therapy reviews, pharmacotherapy consults, anticoagulation management, immunizations, health and wellness programs, and many other clinical services. Reports of pharmacist involvement in community pharmacy based collaborative practice via MTM have also been published. ${ }^{7,23,24}$

$\mathrm{PCMH}$ is an emerging model for organizing and delivering care that has been developed in primary care, particularly in response to a predicted shortage of primary care physicians. It encompasses a team approach to care that may involve various professionals: physicians, advanced practice nurses, physician assistants, nurses, pharmacists, nutritionists, 
social workers, educators, and care coordinators. The model comprises five functions: comprehensive care, patient centeredness, coordination of care, accessible services, and quality and safety. A 2009-2010 report of the American Association of Colleges of Pharmacy identified 151 unique models that define/discuss pharmacy's involvement in primary care services. ${ }^{25}$ The majority of these models focused on chronic disease management (eg, diabetes, hypertension, dyslipidemia), and some specialized services (eg, hepatitis, oncology, tuberculosis, anticoagulation). Most models were situated in ambulatory care settings $(n=81,54 \%)$, while integrated settings $(n=33,22 \%)$, community pharmacy $(n=29$, $19 \%)$, and other $(n=8,5 \%)$ constituted the rest. ${ }^{25}$

The most recent development is that of ACOs which is an organization of health care providers that agrees to be accountable for the quality, cost, and overall care of Medicare beneficiaries who are enrolled in a traditional fee-for-service program who are assigned the ACO."26 The delivery model aims at payment for performance or aligning provider reimbursements to improved quality and reductions in cost of care for patients.

In all these models the essential features are patient centeredness with foundations of communication, health information technology, workforce development, and payment alignment.

\section{Frameworks for CPP}

To reach a higher level and more effective collaboration between practitioners, experience, knowledge, and skills are needed to function in interprofessional health care teams. To accomplish this, collaborative care needs to be emphasized through interprofessional education (IPE) in medical and pharmacy schools. In particular, engaging students into collaborative care in a clinical environment renders great results. $^{27}$

The Interprofessional Education Collaborative Expert Panel 2011, a collaboration of the American Associations of Colleges of Nursing, Pharmacy, Osteopathic Medicine, Medical Colleges, Dental Education, and Schools of Public Health, identified core competencies for interprofessional collaborative practice in four domains: values/ethics, roles/ responsibilities, interprofessional communication, and teams and teamwork. ${ }^{28}$ Their report also outlined desired principles of these competencies; overall, this report serves as the foundational framework for IPE.

One of the challenges of IPE has been encouraging medical students to embrace this concept broadly. Surveys show that pharmacy, nursing, and social work students generally demonstrate a more positive attitude toward IPE than medical students. ${ }^{4}$ One success story is the collaborative venture between the University of Hawaii at Hilo (UHH) College of Pharmacy (CoP) and the John A. Burns School of Medicine Family Medicine Program. ${ }^{29}$ There, the UHH CoP students work with their professors to engage into a collaborative practice with physicians and other medical practitioners. The students use the Hawai' $i$ Island Family Health Center to teach students in various health care disciplines in a collaborative team environment.

Another example is the Inter-Professional Education (IPE) program developed and used by the authors' institution (unpublished data, David Dickter, PhD, 2012). The IPEP curriculum engages health care profession students into clinical studies where students from all programs collaborate through interprofessional teams. Students learn about a variety of issues related to IPE including the formation of an interprofessional team, and the roles and responsibilities of individual team members to promote a culture of safety and to enhance quality of life. The program also allows students to apply the principles they have learned to enhance their clinical practice skills. Initial surveys of students and faculty show strong positive attitudes toward interprofessional collaboration. Other factors that influence establishment of CPP include communication between all stakeholders, team collaboration, attitudes toward such collaboration, training, alignment of payment models, time, and workload..$^{30}$

Whether pharmacists collaborate with physicians within the confines of a practice arrangement in a community pharmacy, a medical office, or in an interprofessional group practice, the first step is to form a successful working relationship. McDonough and Doucette have proposed a model for how pharmacists can form such relationships with physicians. ${ }^{31}$ Within this model, they propose five stages of development toward a collaborative working relationship (CWR) (Figures 1 and 2). As pharmacists and physicians progress through this model, collaboration is theorized to increase and a greater commitment to maintain the relationship is expected to occur, and as each profession learns to depend on the other for input, the relationship is further strengthened. The CWR model, and progression through it, is also influenced by the characteristics of each individual practitioner (eg, age, educational background), the practitioners sites (eg, proximity between), and exchanges between each practitioner. While further research and testing of this specific model is needed, pharmacists should be encouraged to strengthen their relationships with physicians, as well as other providers, toward achieving a successful 


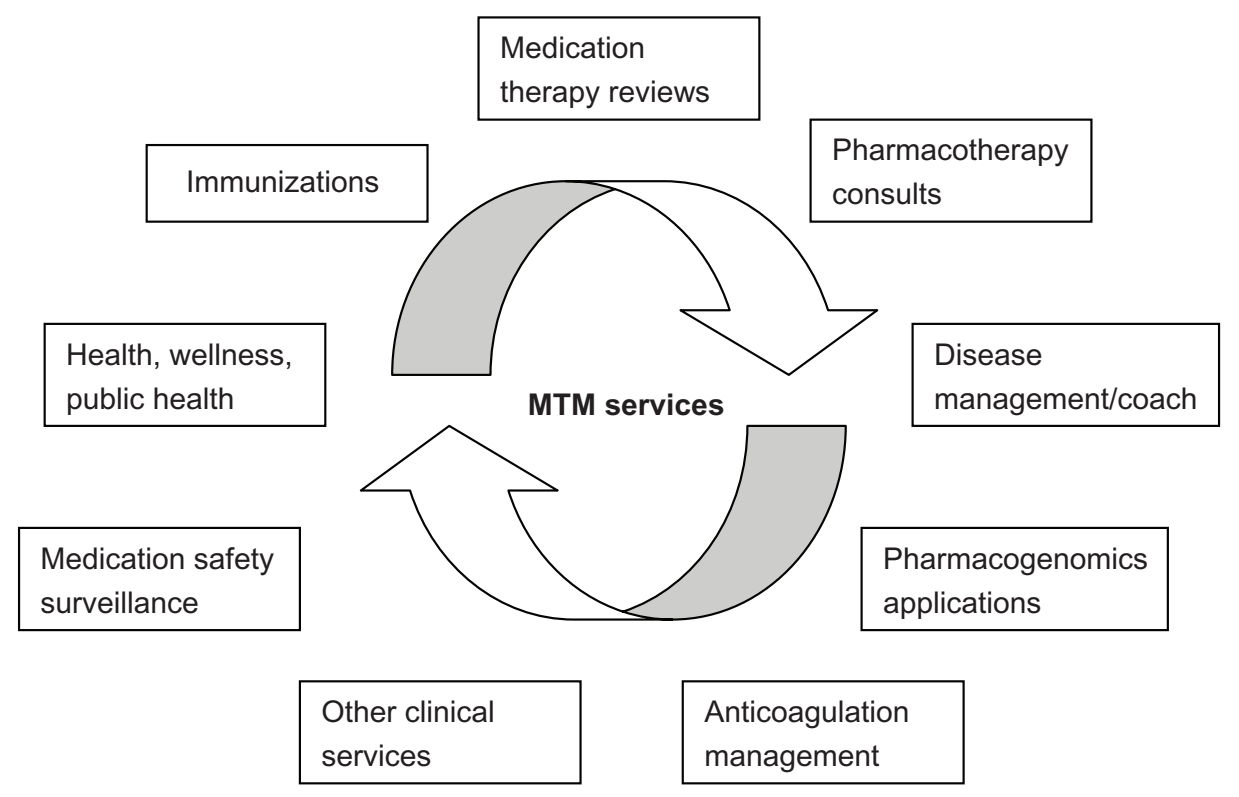

Figure I Spectrum of medication therapy management services.

Notes: $\odot$ Copyright American Pharmacists Association (APhA). Reprinted by permission of APhA. ${ }^{22}$

Abbreviation: MTM, medication therapy management.

CWR leading to positive patient outcomes. Snyder et al used this framework to describe the professional exchanges between pharmacists and physicians engaged in successful CWRs using the Pharmacist-Physician Collaborative Index (PPCI) and qualitative information on relationship initiation, trustworthiness, and role specification..$^{23}$ On the PPCI, both physicians and pharmacists scored similarly on trustworthiness (39.8 \pm 1.7 versus $39.2 \pm 3.1$, respectively); however, physicians scored higher on role specification $(29.8 \pm 2.9$ versus $24.6 \pm 6.9)$ and initiation of the relationship (20.3 \pm 1

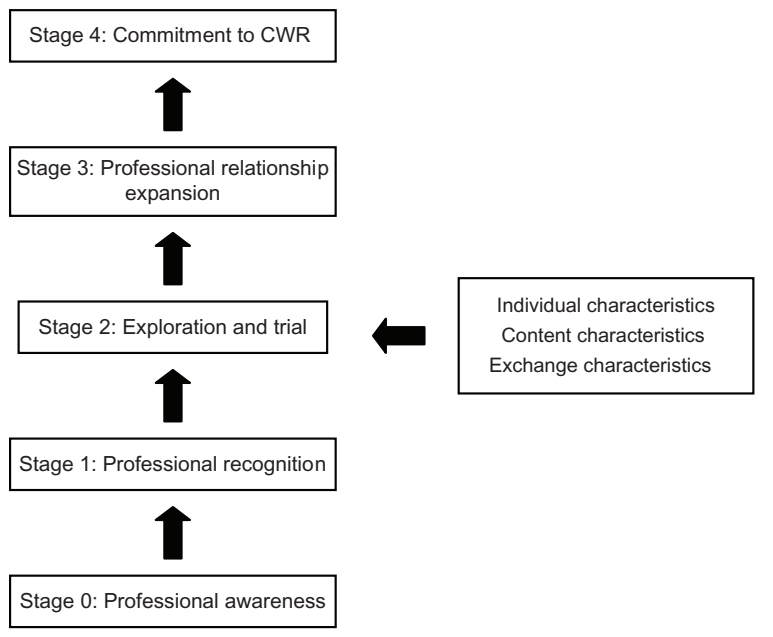

Figure 2 Stepwise approach toward a collaborative working relationship. Notes: Reprinted with permission Watkins J, Landgraf A, Barnett C, Michaud L. Evaluation of pharmacist-provided medication therapy management services in an oncology ambulatory setting. J Am Pharm Assoc (2003). 2012;52: 170-174. ${ }^{32}$ Abbreviation: CWR, collaborative working relationship. versus 16.2 \pm 2.9 ). Qualitatively, it was found that establishing open communication through face to face interactions was important for pharmacists.

\section{Payment models}

One of the challenges to establishing a CPP is the economic justification for the practice model. The establishment of MTM by CMS has provided an avenue for pharmacists to obtain compensation for patient care services in collaborative practice models. In 2005, the American Medical Association supported this opportunity by introducing three Current Procedural Terminology (CPT) codes, specifically for pharmacists to bill for MTM services provided to patients. ${ }^{32}$ However, these codes have not been widely adopted because third party payers do not recognize or compensate pharmacists to bill as independent providers. ${ }^{33}$ Further, pharmacists express dissatisfaction with the amount of compensation provided by the few payers. Therefore, pharmacists have sought other options for billing for these services which include fee for service, incident-to billing, facility fee billing, direct contracting with third party payers, and outcomes based billing. ${ }^{34}$

Incident-to is a billing model where the claim is submitted under the physician's name, with the physician overseeing the work of the pharmacist. This is one way that pharmacists bill Medicare for the services they provide, since Medicare does not allow pharmacists to bill independently under their own name. In order to bill Medicare using incident-to, certain criteria must be met. The pharmacist must be an employee 
of the physician or medical group and while the supervising physician does not have to be in the same room during the visit, the physician must be somewhere on the premises. Most insurance companies will reimburse only a minimal amount for services that are billed using incident-to coding. ${ }^{35}$ Another method of billing insurance companies indirectly for services provided by pharmacists is facility fee billing. A transplant clinic in Spokane, Washington, employed this method by adding the time spent during the pharmacists' services to the facility fee to account for a higher level of facilities used for that patient. ${ }^{35}$ The article did not specify the entities who were billed, but it was reported that pharmacists were responsible for increasing the outpatient reimbursement by approximately $\$ 100$ per patient. $^{35}$

Other practices have successfully contracted directly with third party payers to be reimbursed for their CPP services. Most of these contracted services are reimbursed on a fee for service basis. Towncrest Pharmacy is a community, independent pharmacy that contracted with Iowa State Medicaid to render pharmaceutical case management services, a program that paid pharmacists after each visit for managing the beneficiaries' medications. ${ }^{36}$ Another example is Kerr Drugs stores, a regional pharmacy chain in North Carolina, which contracted with the North Carolina Medicaid Division of Medical Assistance to provide MTM services to their Medicaid beneficiaries. These pharmacists were reimbursed a fixed rate for every medication review that was completed. ${ }^{37}$ Fairview Pharmacy Services established pharmaceutical care services in both Fairview retail pharmacies and primary care clinics to provide MTM services to Minnesota Medicaid beneficiaries. ${ }^{38}$ However, they also offered these services to contracted self-funded employers as well as private pay patients. In this program, the pharmacists were paid for every patient visit with the amount of reimbursement being dependent on the complexity of the patient's case. ${ }^{38}$ While these are examples of fee for service payment models, some practices will contract with payers to get reimbursed on a capitated rate. This occurs particularly with health maintenance organizations which normally pay for services on a per member per month (PMPM) rate.

Pay for performance (P4P) is another business model utilized by health plans to pay provider organizations based on quality performance measures. In California, reimbursement through the P4P program is somewhat of a hybrid between a capitated rate and outcomes based payment. The health plan provides payment to the provider group on a PMPM rate according to their performance. Based on certain established quality measures, if the provider group falls in the 75 th per- centile or higher amongst all the other medical groups, then the health plan will reimburse $100 \%$ of the PMPM rate. The health plan reimbursed $50 \%$ payment for those groups that fell between the 50th and 74th percentiles and minimal to no payment if they were lower than 50th percentile. ${ }^{39}$ Outcomes Pharmaceutical Health Care is an administrative services company that pays pharmacists for MTM services on an outcomes based approach. The company collects fees from health plans or other benefit providers (self-insured employers, union health plans, state Medicaid programs, or Medicare Part D plan sponsors) on a capitated basis and uses those funds to reimburse pharmacists for providing MTM services to benefit enrollees. The network of pharmacies that bill the administrative company for these services include independent, franchise, chain, health system, and consultant pharmacy providers. Payments to pharmacies are processed when claims are submitted by the pharmacists and that are documented in the administrative company's Internet-based documentation system. ${ }^{40}$

In February 2011, an electronic survey was distributed amongst pharmacists to determine what techniques were being employed to bill for outpatient MTM services. Of those reportedly billing for their services, 32 were community pharmacists, 32 were pharmacists billing out of a physician's office, and 31 were pharmacists within a health system outpatient facility. Sixty five percent of the community pharmacists used fee for service, $62 \%$ directly contracted with the third party insurance providers, and $56 \%$ used the pharmacist CPT codes. For those billing out of the physician's office, $71 \%$ used incident-to and $22 \%$ used the pharmacist CPT codes. Almost half of those billing within a health system outpatient facility used facility fee billing, while 36\% used the pharmacist CPT codes, and $29 \%$ used incident-to billing.

\section{Best practices in the US and internationally}

There are many practice models demonstrating collaborative practices. To highlight the best practice models, the literature identified earlier was narrowed down to reflect practice settings that reported positive clinical, humanistic, or financial outcomes. Table 1 provides a layout of the outcomes examined in published studies.

Best practice models are described as either in a community pharmacy setting or in a hospital practice, which includes primary care clinics, nursing facilities, and any other nontraditional community pharmacy setting. The Supplementary material provides a detailed narrative of selected best practices. 


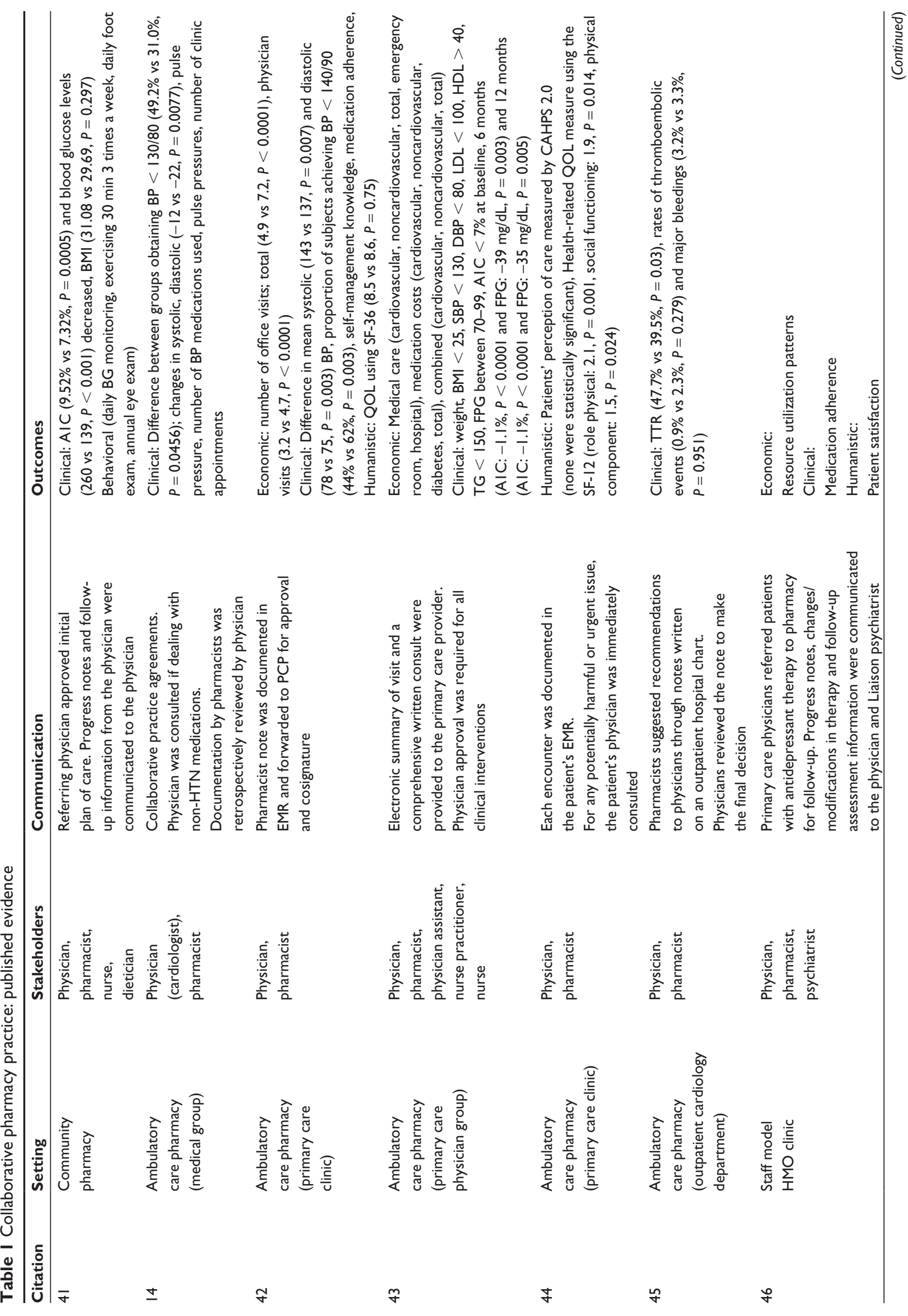




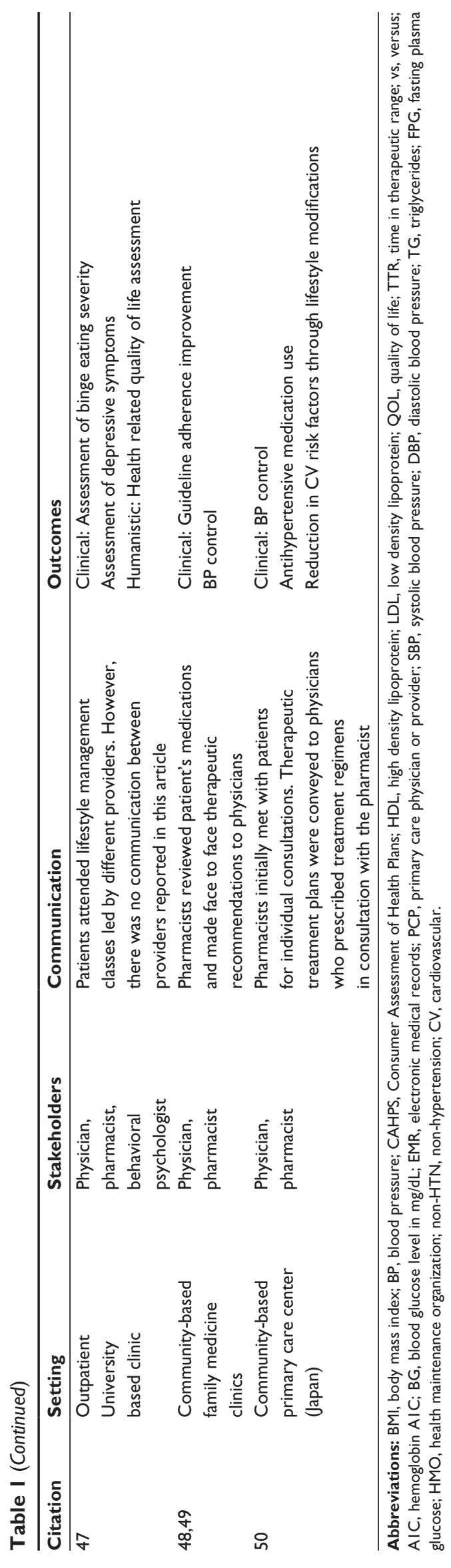

\section{Challenges and barriers in CPP}

Collaborative care is aimed at improving the quality of disease management and standard of health care. As described previously in Figure 2, a good working relationship between pharmacists and other healthcare professionals, especially physicians, is integral in achieving a good corresponding collaborative care model. ${ }^{23,51-55}$ However, some barriers still exist in establishing, developing, and maintaining CPP. These barriers stem from the inherent nature and scope of the pharmacy and medical professions, the working relationship between different health care providers, organizational and financial support, as well as variability in laws and regulations governing CPP. Specifically, these barriers are reported to include boundary or turf concerns, communication breakdowns, power issues, lack of trust, and distance between practice sites. ${ }^{56}$

Traditionally, pharmacists have been typically situated in a physician support role and have not contributed significantly to making major clinical decisions in patient care. Some pharmacists may lack the advanced clinical knowledge and skills in physical examination and patient assessment for providing a full spectrum of patient centered care. As a result, this can be challenging for pharmacists to gain approval from physicians regarding clinical decisions. ${ }^{57}$ This is also an issue in certain countries where the structure of health systems do allow pharmacists to perform clinical services, such as laboratory monitoring or adjustment of the therapeutic regimen for patients.

In addition, practicing pharmacists, especially entry level, may lack confidence in assuming more responsibilities in providing a higher level of patient care as required in collaborative care. Further, although there is interest, health profession education around the globe has not yet placed much stress on IPE in terms of philosophy, academic, or clinical competence. ${ }^{58}$ Lack of available collaborative practice sites for experiential or practical training also limits pharmacist experience and subsequently their level of confidence and comfort in initiating or working when faced with such an environment.

Pharmacist workload is another problem recognized by pharmacists as a barrier to effectively initiating or implementing collaborative care practice with other health care providers. ${ }^{13,51}$ Literature suggests that pharmacist interventions are viewed as extra workload by pharmacists, and potentially cause significant disruption to pharmacists' distributive work schedule and pharmacy service provision. ${ }^{9}$ In addition, settings such as ambulatory care clinics are short staffed, making it difficult for pharmacists to expand their clinical functions in collaborative practice. ${ }^{57}$ 
From a physician perspective, their training since medical school emphasizes their role as leaders who are deemed fully capable in making independent, major, and final patient related decisions in an unidisciplinary health care model ${ }^{58}$ Even though efforts have been made to modify roles to allow engagement of pharmacists in a physician's practice, considerable opposition has been raised from physicians because such reforms are perceived as threats to the independence and autonomy of the physician's practice. ${ }^{10,13,51}$ A difference in attitude toward collaboration has been observed among practitioners and students, where medical students view collaborative practice less favorably than student pharmacists. ${ }^{59} \mathrm{In}$ fact, the above mentioned phenomena are in accordance with the principle of least interest, which proposes that those who are traditionally in a more powerful position are less emotionally invested in expressing eagerness for collaborative relationships with others whom they consider to be lower in the power hierarchy. ${ }^{4}$

Another obstacle in establishing collaborative care practice is to include pharmacists in the physician's daily routine. There is concern whether physicians are able to find time to work with pharmacists in the context of a busy practice. Additionally, the lack of physician experience with clinical pharmacy services and formal collaborative practice legislation were also considered factors that negatively impacted the pharmacist-primary care provider relationship in collaborative care. ${ }^{43}$ In Quebec, Canada, for instance, physicians and pharmacists often do not work in close proximity in primary care settings, and in a majority of the time, telephone conversations and fax transmissions are the only means by which they interact for the exchange of professional opinions and judgments. ${ }^{13}$ In addition, studies in both Canada and in the US suggested that some physicians were not well acquainted with pharmacists' competence in pharmacotherapeutics and ease of access to patients. ${ }^{7}$ With this in mind, physicians may need time to adjust their views on pharmacists, and be ready and generous to fully utilize the skills and expertise of pharmacists to improve health outcomes. On the other hand, with the involvement of pharmacists in collaborative care, some physicians expressed apprehension whether the reduction in the number of medical visits may negatively impact their relationship with patients, and reduce the overall quality of their follow-up. ${ }^{13}$

Regulations are present to build better CPP; although, their establishment also came after a struggle. A study identified the most common barriers in 20 respondent states in the US to pass their original collaborative practice legislations: resistance by physicians or state medical associations (41\%), difficulties in educating health care professionals about pharmacists' abilities (16\%), opposition from pharmaceutical manufacturers $(16 \%)$, opposition from state nursing associations (9\%), and lack of sufficient lobbying efforts among members $(9 \%){ }^{6}$

Effective administrative support, adequate human and financial resources, and continual planned assessment of performance by stakeholders, are all factors necessary for the planning, development, implementation, and success of a collaborative care model. ${ }^{60,61}$ Although collaboration, in general, has been shown to result in cost savings for the health care system through reduction in frequency of hospitalization and more appropriate medication use, in the majority of studies, however, cost effectiveness of the collaborative care model in improving patient outcomes compared to traditional care was not assessed precisely. ${ }^{14,51}$ Every possible effort should be made to determine whether incorporating a medical team with members from different health disciplines is worthwhile by comparing before and after health care expenditure, and improvement in patient outcomes, at individual practice settings. Appropriate and feasible compensation mechanisms to health care providers, including pharmacists, are also essential in encouraging collaboration between health care providers in the organization. ${ }^{13}$ Finally, despite considerable progress in passing and implementing laws on pharmacist collaborative practice, ongoing political uncertainty, which includes opposition from physicians and nurse practitioners, can become a deterrent for advancement of collaborative practice. ${ }^{7,62}$

\section{Looking toward the future of CPP}

With a projected deficiency of physicians estimated to be between 55,000 and 200,000 by 2020 , there is definitely a need for clinical pharmacists to fill the gap through collaborative practice. ${ }^{43}$ To further develop CPP internationally, we need to focus our efforts on education, legislative and regulatory changes, and reimbursement mechanisms.

In 2010, the International Pharmaceutical Federation (FIP) released a policy statement on CPP in which they recommended that each country prepares their pharmacists and health care systems for CPP ${ }^{63}$ The first step in this process is through a fundamental change in pharmacy education to include IPE. ${ }^{58}$ This process will hopefully achieve more positive attitudes toward collaborative practice, a better understanding of each profession's abilities and limitations, and increased communication between disciplines. ${ }^{4,58,59,64}$ Additionally, an important component of 
pharmacy education needs to include developing the skills required to document comprehensive medication reviews, interpret lab results, navigate complex patient situations, and accept clinical uncertainty. These skills will hopefully engender self-confidence in the student pharmacist to take on new responsibilities created by CPP and minimize other traits which would hinder their future ability to succeed in these new roles.$^{64}$ Communication skills will also be a necessary component of pharmacy education to improve interactions with patients, providers, and administrators. ${ }^{5}$

IPE is not limited to the didactic curriculum. One article suggests that if professional students are not able to apply the theory of IPE in an actual environment, then the didactic portion of IPE will be ineffective in the long run. ${ }^{61}$ Academic clinical rotation sites need to adopt CPP models and create those practice environments where student pharmacists can learn how to function in an advanced practice setting and model the behavior shown to them from their preceptors. Education is not limited to student pharmacists, however. Certification or licensing exams will most likely need to demonstrate and ensure the pharmacists' competence to perform in a CPP environment, similar to the Pharmacist Prescriptive Authority Act in New Mexico and the Clinical Pharmacist Practitioner Act in North Carolina. .7,58,65,66 $^{-10}$

The 2010 FIP policy statement also adds legislative and regulatory actions for CPP. It recommends that each country works with their respective governments and health care payers to consider the benefits of CPP to the health care system and provide legislative and financial support to further this practice. ${ }^{63}$ Each country needs to assess their own pharmacy practice laws and strategize the best pathway to expand those laws and regulations to facilitate the development of CPPs that fits with their country's particular health care system. ${ }^{63}$ The US has made some headway in this arena with 46 states having laws and regulations allowing for collaborative drug therapy management between pharmacists and physicians, with New Mexico and North Carolina, as mentioned above, allowing for prescriptive authority for pharmacists through statute. ${ }^{65,67}$ Recently, the US Public Health Service sent a report to the US Surgeon General detailing how pharmacists can improve health care outcomes, relieve demand on the health care system, and reduce costs. ${ }^{68}$ This report led to the US Surgeon General supporting the concept of pharmacists as midlevel providers in the US. These have been large gains, however, the UK is much further along with all pharmacists eligible to obtain prescribing privileges through further training and being recognized as Pharmacist Supplementary Prescribers. ${ }^{65}$ Despite these prescribing powers granted by the government, only a small percentage of pharmacists in the UK have gone through the National Health Service approved training program to acquire these powers. One of the proposed reasons for this phenomenon is difficulty in demonstrating a financial benefit to employers. ${ }^{65}$

As the FIP policy statement noted above, legislative and regulatory involvement also works hand in hand with compensation for clinical services provided. Both are essential to make CPP sustainable. ${ }^{13,65,69-71}$ In the UK they have prescriptive authority granted by legislation but the compensation is not sufficient incentive. In the US we are still working toward having the legislative authority to prescribe and be compensated. In the last few years, three bills have been introduced in the US Congress to allow payments for clinical services and they have failed to get out of the committee level. Thus, a more concerted legislative effort involving pharmacists engaging their locally elected officials is needed to educate them about what clinical pharmacists can do in a CPP model to secure adequate compensation for these services. Recently the Centers for Disease Control and Prevention in the US released a document titled, "Partnering with Pharmacists in the Prevention and Control of Chronic Diseases." ${ }^{" 70}$ In this summary of the available evidence, it states that collaborative drug therapy management brings a return on investment on average of $3: 1$ to $5: 1 .{ }^{70}$ Also, it states that until government and other third party payers realize the value of CPP services, the best partners in the meantime are academic institutions and self-insured employers (companies that assume the health risk of their employees and other beneficiaries). ${ }^{70}$ Thus, we can continue our CPP models with these partners and further our research in this area to create a more compelling case for higher level decision makers.

\section{Summary}

The time is ripe for interprofessional collaborative practice to become a reality that all health professions commit to given the evidence that such practice has the potential to positively impact continuity and coordination of care to improve medication adherence, reduce adverse events and errors, ultimately improve patient health outcomes, and reduce economic burden. Although regulation and education have bought in to the concept of collaborative practice, and various examples are in existence, barriers such as resistance from professionals, logistic issues, and lack of adequate payment mechanisms continue to impact the expansion of CPP. A combination of approaches, from education, regulation, and health systems, is needed to examine the evidence and the infrastructure to make collaborative practice a feasible model of care. 


\section{Disclosure}

The authors declare no conflicts of interest in this work.

\section{References}

1. Gladwell M. The Tipping Point: How Little Things can make a Big Difference. New York: Little Brown; 2000.

2. Kohn LT, Corrigan JM, Donaldson MS, editors. To Err is Human: Building a Safer Health System. Washington: National Academy Press; 2000.

3. Committee on Quality of Health Care in America. Crossing the Quality Chasm: A New Health System for the 21st Century. Washington: National Academy Press; 2001.

4. Van Winkle L, Fjortoft N, Hojat M. Validation of an instrument to measure pharmacy and medical students' attitudes toward physicianpharmacist collaboration. Am J Pharm Educ. 2011;75(9):1-6.

5. Levenson S, Saffel D. The consultant pharmacist and the physician in the nursing home: roles, relationships, and a recipe for success. $J \mathrm{Am}$ Med Dir Assoc. 2007;8:55-64.

6. Punekar Y, Lin SW, Thomas J III. Progress of pharmacist collaborative practice: status of state laws and regulations and perceived impact of collaborative practice. J Am Pharm Assoc (2003). 2003;43:503-510.

7. Alkhateeb F, Unni E, Latif D, Shawaqfeh M, Al-Rousan R. Physician attitudes toward collaborative agreements with pharmacists and their expectations of community pharmacists' responsibilities in West Virginia. J Am Pharm Assoc (2003). 2009;49:797-800.

8. Ukens C. Most states now allow collaborative practice. [webpage on the Internet]. Drug Topics. Available from: http://drugtopics. modernmedicine.com/drugtopics/Community+Pharmacy/Moststates-now-allow-collaborativepractice/ArticleStandard/Article/ detail/359816. Accessed December 6, 2012.

9. McKinnon A, Jorgenson D. Pharmacist and physician collaborative prescribing: for medical renewals within a primary health centre. Can Fam Physician. 2009;55:e86-e91.

10. Pottie K, Farrel B, Haydt S, et al. Integrating pharmacists into family practice teams: physicians' perspectives on collaborative care. Can Fam Physician. 2008;54:1714-1717. e5.

11. Hammad EA, Yasein N, Tahaineh L, Albsoul-Younes A. A randomized controlled trial to assess pharmacist-physician collaborative practice in the management of metabolic syndrome in a university medical clinic in Jordan. J Manag Care Pharm. 2011;17:295-303.

12. Mino-Leon D, Reyes-Morales H, Jasso L, Doubova SV. Physicians and pharmacists: collaboration to improve the quality of prescriptions in primary care in Mexico. Int J Clin Pharm. 2012;34:475-480.

13. Lalonde L, Hudon E, Goudreau J, et al. Physician-pharmacist collaborative care in dyslipidemia management: The perception of clinicians and patients. Res Soc Adm Pharm. 2011;7:233-245.

14. Irons BK, Meyerrose G, Laguardia S, Hazel K, Seifert CF. A collaborative cardiologist-pharmacist care model to improve hypertension management in patients with or at high risk for cardiovascular disease. Pharm Prac. 2012;10:25-32.

15. Carter BL, Clarke W, Ardery G, et al; Collaboration Among Pharmacists Physicians To Improve Outcomes Now (CAPTION) Trial Investigators. A cluster-randomized effectiveness trial of a physician-pharmacist collaborative model to improve blood pressure control. Circ Cardiovasc Qual Outcomes. 2010;3:418-423.

16. St Peter WL, Farley TM, Carter BL. Role of collaborative care models including pharmacists in improving blood pressure management in chronic kidney disease patients. Curr Opin Nephrol Hypertens. 2011;20: 498-503.

17. Kucukarslan S, Lai S, Dong Y, Al-Bassam N, Kim K. Physician beliefs and attitudes towards collaboration with community pharmacists. Res Soc Admin Pharm. 2011;7:224-232.

18. Gurnee MC, DaSilva RV. Constructing Disease Management Programs. [webpage on the Internet]. Managed Care; 2007. Available from: http:/ www.managedcaremag.com/archives/9706/9706.disease_man.shtml. Accessed January 20, 2013.
19. Disease Management Association of America. Definition of Disease Management. Available from: http://www.dmaa.org/definition.html. Accessed January 22, 2013.

20. Bunting BA, Smith BH, Sutherland SE. The Asheville Project: Clinical and economic outcomes of a community-based long-term medication therapy management program for hypertension and dyslipidemia. J Am Pharm Assoc (2003). 2008;48:23-31.

21. Garrett DG, Bluml BM. Patient self-management program for diabetes: first-year clinical, humanistic, and economic outcomes. J Am Pharm Assoc (2003). 2005;45:130-137.

22. American Pharmacists Association [webpage on the Internet]. What is Medication Therapy Management? Available from: http://www. pharmacist.com $/ \mathrm{mtm}$. Accessed December 10, 2012.

23. Snyder ME, Zillich AJ, Primack BA, et al. Exploring successful community pharmacist-physician collaborative working relationships using mixed methods. Res Soc Adm Pharm. 2010;6:307-323.

24. Bryant LJM, Coster G, Gamble GD, McCormick RN. The General Practitioner-Pharmacist Collaboration (GPPC) Study: a randomized controlled trial of clinical medication reviews in community pharmacy. Int J Pharm Pract. 2011;19:94-105.

25. Haines SL, DeHart RM, Hess KM, et al; Professional Affairs Committee. Report of the 2009-2010 Professional Affairs Committee: pharmacist integration in primary care and the role of academic pharmacy. Am J Pharm Educ. 2010;74:S5.

26. Medicare "Accountable Care Organizations" Shared Savings Program - New Section 1899 of Title XVIII, Preliminary Questions and Answers". Centers for Medicare and Medicaid Services. Available from: http://www.healthcare.gov/news/factsheets/2011/03/accountablecare03312011a.html. Accessed April 20, 2013.

27. Arredondo A, Henderson B, Blackburn W, Klotz R. An Advanced Pharmacy Practice Rotation in a Medical Group Practice: A Pharmacy Student's Perspective. California DO Fall 2012. Available from: ws.westernu.edu/western-News/docs/CA-DO-1.pdf. Accessed April 15, 2013.

28. Interprofessional Education Collaborative Expert Panel. (2011). Core Competencies for Interprofessional Collaborative Practice: Report of an Expert Panel. Washington, DC: Interprofessional Education Collaborative. Available from: http://www.aacn.nche.edu/educationresources/ipecreport.pdf. Accessed January 25, 2013.

29. Ma C, Holuby R, Bucci L. Physician and pharmacist collaboration: the University of Hawaii at Hilo college of pharmacy - JABSOM experience. Hawaii Med J. 2010;69:42-44.

30. O'Daniel M, Rosenstein AH. Professional Communication and Team Collaboration. In: Hughes RG, editor. Patient Safety and Quality: An Evidence-Based Handbookfor Nurses. Rockville: Agency for Healthcare Research and Quality (US); 2008:Chapter 33. Available from: http:// www.ncbi.nlm.nih.gov/books/NBK2637/. Accessed April 15, 2013.

31. McDonough RP, Doucette WR. Developing collaborative working relationships between pharmacists and physicians. J Am Pharm Assoc. 2001;41(5):682-692.

32. Watkins J, Landgraf A, Barnett C, Michaud L. Evaluation of pharmacist-provided medication therapy management services in an oncology ambulatory setting. $J$ Am Pharm Assoc (2003). 2012;52: $170-174$.

33. Scott MA, Hitch WJ, Wilson CG, Lugo AM. Billing for pharmacists' cognitive services in physicians' offices: Multiple methods of reimbursement. J Am Pharm Assoc (2003). 2012;52:175-180.

34. Beatty SJ, McCormick KM, Beale DJ, et al. Current trends in outpatient pharmacy services and billing. J Am Pharm Assoc (2003). 2012;52:154-160.

35. Maldonado AQ, Seiger TC, Urann CL, McCleary JA, Goroski AL. Ojogho ON. Billing for outpatient transplant pharmacy services. Am J Health Sys Pharm. 2012;69:144-147.

36. McDonough RP, Harthan AA, McLeese KE, Doucette WR. Retrospective financial analysis of medication therapy management services from the pharmacy's perspective. J Am Pharm Assoc (2003). 2010;50: $62-66$. 
37. Michaels NM, Jenkins GF, Pruss DL, Heidrick JE, Ferreri SP. Retrospective analysis of community pharmacists recommendations in the North Carolina Medicaid medication therapy management program. J Am Pharm Assoc (2003). 2010;50:347-353.

38. Oliveria DJ, Brummel AR, Miller DB. Medication therapy management: 10 years of experience in a large integrated health care system. J Manag Care Pharm. 2010;16:185-195.

39. Cutler T, Palmieri J, Khalsa M, Stebbins M. Evaluation of the relationship between a chronic disease care management program and California pay-for-performance diabetes care cholesterol measures in one medical group. J Manag Care Pharm. 2007;13:578-588.

40. Barnett M, Frank J, Wehring H, et al. Analysis of pharmacist-provided medication therapy management (MTM) services in community pharmacies over 7 years. J Manag Care Pharm. 2009;15:18-31.

41. West D, Blevins MA, Brech D, Stotts F, Gardner S. A multidisciplinary approach in a community pharmacy can improve outcomes for diabetes patients. Diabetes Educ. 2003;29:962-968.

42. Hunt JS, Siemenczuk J, Pape G, et al. A randomized controlled trial of team-based care: impact of physician-pharmacist collaboration on uncontrolled hypertension. J Gen Intern Med. 2008;23(12): 1966-1972.

43. Monte SV, Slaza EM, Albanese NP, Adelman M, Rao G, Paladino JA. Clinical and economic impact of a diabetes clinical pharmacy service program in a university and primary care-based collaboration model. J Am Pharm Assoc (2003). 2009;49:200-208.

44. Isetts B, Schondelmeyer S, Heaton A, Wadd WB, Hardie NA, Artz MB. Effects of collaborative drug therapy management on patients' perceptions of care and health-related quality of life. Res Social Adm Pharm. 2006;2:129-142.

45. Saokaew S, Sapoo U, Nathisuwan S, Chaiyakunapruk N, Permsuwan U. Anticoagulation control of pharmacist-managed collaborative care versus usual care in Thailand. Int J Clin Pharm. 2012;34:105-112.

46. Finley P, Rens H, Pont J, et al. Impact of a collaborative pharmacy practice model on the treatment of depression in primary care. Am J Health Syst Pharm. 2002;59:1518-1526.

47. Malone M, Alger-Mayer S, Anderson D. The lifestyle challenge program: a multidisciplinary approach to weight management. Ann Pharmacother. 2005;39:2015-2020.

48. Carter B, Ardery G, Dawson J, et al. Physician and pharmacist collaboration to improve blood pressure control. Arch Intern Med. 2009; 169:1996-2002.

49. Weber C, Ernst M, Sezate G, Zheng S, Carter B. Pharmacist-physician comanagement of hypertension and reduction in 24-hour ambulatory blood pressures. Arch Intern Med. 2010;170:1634-1639.

50. Tobari H, Arimoto T, Shimojo N, et al. Physician-pharmacist cooperation program for blood pressure control in patients with hypertension: a randomized-controlled trial. Am J Hypertens. 2010;23:1144-1152.

51. Dey RM, de Vries MJW, Bosnic-Anticevich S. Collaboration in chronic care: unpacking the relationship of pharmacists and general medical practitioners in primary care. Int J Pharm Pract. 2011;19:21-29.

52. Brock KA, Doucette WR. Collaborative working relationships between pharmacists and physicians: an exploratory study. J Am Pharm Assoc (2003). 2004;44:358-365.

53. Mehta BH, Snyder ME, Nikitas A. Developing collaborative relationships between pharmacists and other health professionals. $J \mathrm{Am}$ Pharm Assoc (2003). 2011;51:332-338.

54. Liu Y, Doucette W, Farris K. Examining the development of pharmacist-physician collaboration over 3 months. Res Social Adm Pharm. 2010;6:324-333.
55. Liu Y, Doucette W. Exploring stages of pharmacist-physician collaboration using the model of collaborative working relationship. J Am Pharm Assoc (2003). 2011;51:412-417.

56. Miccolo MA, Spanier AH. Critical care management in the 1990s. Making collaborative practice work. Crit Care Clin. 1993;9:443-453.

57. Edwards J, Edwards S, Saltman D. The role of the nurse practitioner and clinical pharmacist in collaborative patient care and drug therapy management in Canadian cancer centres. Report Card on Cancer in Canada, 2010-2011. 2010;13:30-33.

58. Ray M. Shared borders: Achieving the goals of interdisciplinary patient care. Am J Health Syst Pharm. 1998;55:1369-1374.

59. Van Winkle L, Bjork B, Chandar N, et al. Interprofessional workshop to improve mutual understanding between pharmacy and medical students. Am J Pharm Educ. 2012;76:150.

60. Warrington L, Ayers P, Baldwin A, et al. Implementation of a pharmacistled, multidisciplinary diabetes management team. Am J Health Syst Pharm. 2012;69:1240-1245.

61. Drummond N, Abbott K, Williamson T, Somji B. Interprofessional primary care in academic family medicine clinics. Can Fam Physician. 2012;58:e450-e458.

62. Thompson CA. Collaborative practice comes to New York, expands in Indiana. AJHP News. July 15, 2011.

63. FIP Statement of Policy on Collaborative Pharmacy Practice FIP, 2010. Available from: http://www.fip.org/statements. [homepage on the Internet]. Accessed April 15, 2013.

64. Farrell B, Ward N, Dore N, Russel G, Geneau R, Evans S. Working in interprofessional primary health care teams: what do pharmacists do? Res Social Adm Pharm. 2012;8:1-14.

65. Murawski M, Villa KR, Dole EJ, et al. Advanced-practice pharmacists: practice characteristics and reimbursement of pharmacists certified for collaborative clinical practice in New Mexico and North Carolina. Am J Health Syst Pharm. 2011;68:2341-2350.

66. Martin-Boone JE, Pruce D, Airaksinen M, et al. FIP reference paper collaborative practice. 2009:1-76. Available from: http://www.fip.org/ statements. [homepage on the Internet]. Accessed April 15, 2013.

67. Academy of Managed Care Pharmacy. Practice Advisory on Collaborative Drug Therapy Management. Academy of Managed Care Pharmacy; 2012. Available from: http:/www.amcp.org/WorkArea/ DownloadAsset.aspx?id=14710. Accessed December 11, 2012

68. Giberson S, Yoder S, Lee MP. Improving Patient and Health System Outcomes through Advanced Pharmacy Practice. A Report to the US Surgeon General. Office of the Chief Pharmacist. US Public Health Service; 2011.

69. Moore A, Patterson C, White J, et al. Interprofessional and integrated care of the elderly in a family health team. Can Fam Physician. 2012;58:e436-e441.

70. Morrison CM, Glover D, Gilchrist SM, et al. Partnering with Pharmacists in the Prevention and Control of Chronic Diseases. Atlanta: Centers for Disease Control and Prevention; 2012. Available from: http:// www.cdc.gov/dhdsp/programs/nhdsp_program/docs/pharmacist_guide. pdf. Accessed April 15, 2013.

71. Howard M, Trim K, Woodward C, et al. Collaboration between community pharmacists and family physicians: lessons learned from the seniors medication assessment research trial. J Am Pharm Assoc (2003). 2003;43:566-572. 


\section{Supplementary material \\ Community pharmacy}

AllCare Pharmacy in Arkadelphia, Arkansas, developed a diabetes self-management training program according to the National Standards for Diabetes Self-Management Education, which is published by the American Diabetes Association. The program included a physician, pharmacist, nurse, and dietician. The pharmacist was a full time employee of the pharmacy and the physician served as a consultant, with all the other employees only paid part time. Marketing the service to physicians and nurses included in-person visits and telephone calls. The program was designed using the Medicare model where the initial assessment is done one on one followed by eight weekly group education sessions, each lasting about 60 minutes. Patients were able to visit the pharmacy any time they had questions or concerns since the pharmacist was accessible in the pharmacy during business hours. This study offered some evidence that providing diabetes education in a community pharmacy is effective at improving intermediate clinical outcomes. There was an average significant reduction in hemoglobin A1C (A1C) by 2.2 percentage points $(P=0.0005)$ and a decrease in glucose levels (from $260 \mathrm{mg} / \mathrm{dL}$ to $139 \mathrm{mg} / \mathrm{dL}, P<0.001$ ), but body mass index did not change significantly. There was also an improvement in self-care behaviors which can lead to a reduction in the risk of diabetes complications. ${ }^{1}$

\section{Hospital practice}

Hospital practice sites in the US that demonstrate best practice models include a collaborative practice model at Kaiser Permanente, (Oakland, CA, USA), a primary care facility within a large nonprofit staff model health maintenance organization (Kaiser Permanente of Northern California). ${ }^{2}$ In this practice site, pharmacists provided medication therapy management for patients who were taking psychiatric medications such as antidepressants. A cohort of 13 physicians referred patients who started on antidepressants to the practice model. Clinical pharmacy specialists coordinated the follow-up with patients through office visits and telephone calls. Pharmacists had limited prescribing privileges to allow for dose adjustments and to add on ancillary medications as deemed necessary. Pharmacists, physicians, and psychiatrists met regularly to discuss patients' progress. Patients' adherence to treatment, satisfaction, and costs to the health maintenance organization (HMO) were compared to a control group of patients being treated by other physicians in the practice. This multidisciplinary model was associated with a significant improvement in medication adherence rates (the medication possession ratio was 0.81 for the intervention group versus 0.66 for the control group) in comparison to patients receiving usual care. It also reduced visits to the primary care physician (39\% for the control group versus $12 \%$ for the intervention group, $P=0.029$ ).

The Lifestyle Challenge Program at a facility in New York presented results in 2005 of a multidisciplinary weight management program incorporating diet, lifestyle, physical activity, and behavioral modification. ${ }^{3}$ The goal of the program was to educate patients using behavioral modification techniques to improve health and weight loss. Participants were required to attend 1 hour group based sessions over a 20 week period. Sessions were led by a facilitator with expertise on the topic being discussed. Participants were only educated on weight reducing diets, but were not required to follow a specific dietary plan. Facilitators included a physician specializing in nutrition, a pharmacist, a dietician or exercise physiologist, and a behavioral psychologist. Ninety participants who had multiple obesity related comorbid diseases entered the program. The 39 patients who completed the study showed significant improvement in health related quality of life, binge eating behavior, and depressive symptoms.

\section{Ambulatory care settings}

A prospective, cluster randomized controlled clinical trial at six community based family medicine residency programs in Iowa evaluated a physician-pharmacist collaborative model to improve blood pressure control. ${ }^{4,5}$ The study enrolled 402 patients with uncontrolled hypertension. Clinical pharmacists made drug therapy recommendations to physicians during office visits with the patients. Physicians and pharmacists worked together to decide how best to implement the proposed interventions. Adherence to national guidelines was improved in the intervention group compared to the control group (the difference between baseline and a 6 month followup for the mean adherence score was an $8.1 \%$ increase for the control group and a $55.4 \%$ increase for the intervention group, $P=0.09$ ). The mean blood pressure decreased by $6.8 / 4.5 \mathrm{mmHg}$ in the control group and 20.7/9.7 $\mathrm{mmHg}$ in the intervention group $(P=0.05$ for between group systolic blood pressure comparison). Blood pressure was controlled in $29.9 \%$ of patients in the control group and in $63.9 \%$ of patients in the intervention group $(P=0.001)$.

A collaborative physician-pharmacist model to improve diabetes outcomes was initiated in a multispecialty managed care physician group practice. ${ }^{6}$ In this model, 22 physicians held practice agreements with two pharmacy specialists 
allowing the pharmacists to initiate, adjust, or discontinue medications used for the treatment of diabetes, dyslipidemia, and hypertension based on an approved protocol. Standing order protocols were in place to allow laboratory tests to be ordered and referrals to be made. Clinical outcomes measured included A1C, lipids, and frequency of adherence to preventative care (annual foot and eye examinations and daily aspirin therapy). The model showcased that pharmacist-coordinated diabetes management improved clinical markers; there were significant improvements in A1C reduction and low density lipoprotein levels, as well as the frequency of adherence to preventative care.

In 2007, The Texas Tech University Health Sciences Center's University Cardiology Group was composed of four full time cardiologists, with only one of these physicians participating in a collaborative practice model. This cardiologist referred his patients with hypertension to the hypertension service, which was staffed by two clinical pharmacists, at his discretion. Collaborative practice agreements were utilized to allow the pharmacists to adjust the patients' drug therapies on behalf of the physician and to schedule follow-up appointments as needed. The pharmacists did not use a specific formulary or algorithm, but made clinical decisions on a case by case basis. The collaborating cardiologist would be consulted if recommendations were made on nonhypertension medications or for issues outside the pharmacists' scope of practice. Patients were discharged back to usual care once the patient's blood pressure was under control for at least two follow-up visits and maintained for several months. The cardiology group conducted a study comparing the quality of care of a physician-pharmacist collaborative practice model to usual care with no clinical pharmacy intervention in patients with hypertension in the same clinical setting. Significant improvements from baseline in systolic blood pressure, diastolic blood pressure, and pulse pressure were observed in the pharmacist-physician care model. When compared to usual care, the physicianpharmacist model was more likely to get patients to an aggressive goal blood pressure. While the total duration of follow-up was shorter in the collaborative practice care model, the frequency of clinic visits and blood pressure assessments were higher in this model. ${ }^{7}$

The Providence Primary Care Research Network in Oregon is comprised of nine community based primary care clinics with approximately 80 physicians providing care to 110,000 patients. The collaborative practice model of this network consisted of five pharmacy practitioners. Each pharmacist had one or two years of ambulatory care residency training and was board certified in pharmacotherapy. Initial visits with the patients included a description by the pharmacist of the physician-pharmacist collaborative model of care. The pharmacists followed Network approved collaborative hypertension management guidelines to review and adjust patients' medication therapies. Each pharmacist had access to a patient's medical records as well as access to the primary care physician to discuss treatment plans or other medical issues as needed. Notes were documented in the electronic medical record and forwarded to the appropriate physician for approval and cosignature. In this study, subjects cared for in the physician-pharmacist team model were $40 \%$ more likely to achieve their goal blood pressure compared to those cared for by their physician alone. The collaborative practice model showed an increase in total office visits, with a significant decrease in the number of physician visits.

Although this study did not assess patient medication cost share, the higher generic prescribing rate in the collaborative arm could be considered a surrogate outcome for cost. However, there were minimal differences between groups in hypertension-related knowledge, medication adherence, quality of life, or patient satisfaction. ${ }^{8}$

Fairview Health Services of Minnesota-St Paul, Minneapolis, established a Collaborative Drug Therapy Management (CDTM) model in six primary clinics called the Collaborative Practice of Pharmaceutical Care. Since its inception in 1999, the Fairview CDTM has shown improvements in patients' goals of therapy and has accounted for the identification and resolution of more than 12,000 drug therapy problems. The pharmacists involved in the program follow a systematic patient care process that includes assessing all of a patient's medication therapy needs, designing a pharmaceutical care plan, and conducting follow-up evaluations to determine outcomes. Therapeutic goals for each patient included clinical goals following evidence in the literature or national treatment guidelines, as well as practical and measurable patient-specific goals. For any potentially harmful or urgent issues, the patient's physician was immediately contacted. Patient perceptions of care were slightly higher for those who received CDTM services compared to those who did not, but this was not statistically significant. Health related quality of life, assessed using the short-form-12 instrument, showed significant improvement in the CDTM intervention. ${ }^{9}$

\section{Academic partnerships}

The MedSense Program was developed by the University of Buffalo School of Pharmacy and Pharmaceutical 
Sciences in collaboration with a regional primary care physician group. Patients with type 2 diabetes and seen by the physician group were identified by either a physician, physician assistant, nurse practitioner, pharmacist, or nurse and offered a consultation with the MedSense pharmacist. The MedSense pharmacist was either a clinical assistant professor of pharmacy with a residency in ambulatory care and with board certification as a pharmacotherapy specialist, or a postgraduate year 1 pharmacy practice resident. Neither pharmacist had any additional diabetes-specific training. At the initial visit, pharmacists assessed the medication therapy and then provided the primary care provider with a brief electronic summary and a comprehensive written consult. Since New York state legislation does not allow for collaborative practice agreements between pharmacists and physicians for pharmacotherapy management, physician approval was required for all clinical interventions. Patients enrolled in the program experienced significant reductions in A1C $(-1.1 \%, P<0.00001)$ and fasting plasma glucose $(-39 \mathrm{mg} / \mathrm{dL}, P<0.03)$ from baseline after 6 months. However, low density lipoprotein, triglycerides, blood pressure, weight, and body mass index were reduced but not statistically significant. Also, there was not much difference in any of the clinical outcomes between 6 months and 12 months. While there were a number of patients whose metabolic parameters improved, there was no difference for patients whose metabolic parameters were at goal between baseline and 6 and 12 months. Out of 184 pharmacologic recommendations, $45 \%$ were accepted, $30 \%$ were declined, and $25 \%$ were unable to be determined due to lack of documentation. Medical costs tended to decrease while prescription medication costs tended to increase, but these trends were not statistically significant. ${ }^{10}$

\section{Examples across the globe}

Internationally, the following models demonstrate best practice as seen in the literature. At the Mahart Nakornratchasima Hospital's Department of Outpatient Cardiology and Cardiovascular Thoracic Surgery in Thailand, a pharmacist-managed warfarin therapy collaborative care model was established. Two clinical pharmacists were trained through structured didactic teaching, as well as with practice sessions with case studies for 2 months under a senior clinical pharmacist who had experience providing anticoagulation therapy management for over 7 years. The clinical pharmacists met one on one with patients receiving warfarin therapy to identify any warfarin-related issues and then made recommendations to the patient's physician in the outpatient hospital chart. Physicians then made the final decisions regarding the patient's therapy after reviewing the notes. The hospital conducted a study comparing the collaborative care model versus usual care without intensive pharmacist intervention. Patients spent more time in therapeutic range under the collaborative care model than those patients under usual care $(47.7 \%$ versus $39.5 \%$, $P=0.003$ ) with no difference in bleeding events between the two groups. The clinical pharmacists made 284 interventions over a 9 month period and $228(80 \%)$ of them were accepted by the physicians. ${ }^{11}$

A study conducted in Japan highlights a collaborative practice between physicians and pharmacists in the role of managing hypertension. ${ }^{12}$ This 6 -month randomized controlled trial for blood pressure control was conducted at a community based primary care center. Patients in the intervention group received 15 minute sessions of individual counseling monthly for 6 months with a pharmacist. The pharmacist made lifestyle recommendations at each visit, and offered the physician therapeutic recommendations on the patient's antihypertensive medications. Physicians and pharmacists discussed treatment plans for the patient either via phone or face to face. The mean decrease in SBP/DBP was $2.9 / 3.3 \mathrm{mmHg}$ in the intervention group over baseline ( $P=0.02$ and $P<0.00001$ for SBP and DBP, respectively). A significantly higher percentage of patients in the intervention group were able to reduce the use of antihypertensive medications $(P<0.0001)$.

\section{A personal example}

Roger Klotz and Micah Hata initiated an Anticoagulation Clinic in a community pharmacy in 2009. A collaborative practice protocol was created and a physician agreed to review the protocol and sign off that it was medically appropriate. The protocol requires that a referral form be completed and signed by the referring physician and faxed to the community pharmacy. The protocol allows the pharmacist to manage the therapy which includes determining the INR using a point of care device, making a dosage adjustment when necessary, and determining the next clinic visit/ appointment. The written progress note including the INR, dosage adjustment, next visit, and any observations made by the pharmacist is then faxed to the referring physician. The clinic has been managing many of the patients for 3.5 years with no difficulties. Initially, the authors thought that the major barrier to implement this collaborative would be physicians' concern about a community pharmacist managing a very difficult therapy, as well as patient resistance to having a 
pharmacist managing their therapy. These turned out to not be barriers at all. The major barrier to implementation of a community pharmacy managed anticoagulation clinic was the payers. Medicare Intermediate stated that pharmacists are not approved as a provider under Medicare so this would eliminate a major patient population. The authors then focused on the private payers which included Medicare Part C payers. Under Medicare Part C, providers do not bill Medicare and they must bill the Part $\mathrm{C}$ payer. Nonmedicare patients, of course, have private payers purchased by themselves or their employer so that would allow the pharmacist managed anticoagulation clinic to bill these payers. Therapy management must be billed to the patient's Major Medical Plan so all billing must be done via the Form 1500 form. The major payers require claims to be submitted electronically. Fortunately, there are a number of organizations that have systems that allow practitioners to submit electronic claims. We chose an appropriate medical claims organization and began using their Web based system. In order to submit claims you must provide the diagnosis (International Classification of Disease [ICD]-9 code) that requires the interventions, the appropriate current procedural terminology (CPT) code(s), the referring physicians NPI number, the pharmacist provider's national provider identification (NPI) number, and the NPI number for the organization receiving payments. Our referral form requires the referring physician to provide the diagnosis and appropriate ICD-9 code so this assures the payer that there is a medical need for the intervention. Pharmacists must provide the appropriate CPT codes for the INR test and the clinic visit. Initially, the payers were hesitant to pay pharmacists for managing anticoagulation therapy. The major payer in California was hesitant, and emailed questions over approximately 4 months which the anticoagulation clinic pharmacists responded to. Finally, we were notified that the payer had put the clinic into their computer as an approved provider. Claims are paid electronically within 30 to 45 days of submission. We are now receiving reimbursement from a number of Californian payers which include payment of claims for Medicare patients who have a preferred provider organization under Part C. It does take time and effort to become a provider that is approved to submit claims and receive payment.

\section{References}

1. West D, Blevins MA, Brech D, Stotts F, Gardner S. A multidisciplinary approach in a community pharmacy can improve outcomes for diabetes patients. Diabetes Educ. 2003;29:962-968.

2. Finley $\mathrm{P}$, Rens $\mathrm{H}$, Pont $\mathrm{J}$, et al. Impact of a collaborative pharmacy practice model on the treatment of depression in primary care. Am J Health Syst Pharm. 2002;59:1518-1526.

3. Malone M, Alger-Mayer S, Anderson D. The lifestyle challenge program: a multidisciplinary approach to weight management. Ann Pharmacother. 2005;39:2015-2020.

4. Carter B, Ardery G, Dawson J, et al. Physician and pharmacist collaboration to improve blood pressure control. Arch Intern Med. 2009; 169:1996-2002.

5. Weber C, Ernst M, Sezate G, Zheng S, Carter B. Pharmacist-physician comanagement of hypertension and reduction in 24-hour ambulatory blood pressures. Arch Intern Med. 2010;170:1634-1639.

6. Kiel P, McCord A. Pharmacist impact on clinical outcomes in a diabetes disease management program via collaborative practice. Ann Pharmacother. 2005;39:1828-1832.

7. Irons BK, Meyerrose G, Laguardia S, Hazel K, Seifert CF. A collaborative cardiologist-pharmacist care model to improve hypertension management in patients with or at high risk for cardiovascular disease. Pharm Prac. 2012;10:25-32.

8. Hunt JS, Siemenczuk J, Pape G, et al. A randomized controlled trial of team-based care: impact of physician-pharmacist collaboration on uncontrolled hypertension. J Gen Intern Med. 2008;23(12): 1966-1972.

9. Isetts B, Schondelmeyer S, Heaton A, et al. Effects of collaborative drug therapy management on patients' perceptions of care and health-related quality of life. Res Social Adm Pharm. 2006;2:129-142.

10. Monte SV, Slaza EM, Albanese NP, Adelman M, Rao G, Paladino JA. Clinical and economic impact of a diabetes clinical pharmacy service program in a university and primary care--based collaboration model. J Am Pharm Assoc (2003). 2009;49:200-208.

11. Saokaew S, Sapoo U, Nathisuwan S, Chaiyakunapruk N, Permsuwan U. Anticoagulation control of pharmacist-managed collaborative care versus usual care in Thailand. Int J Clin Pharm. 2012;34:105-112.

12. Tobari H, Arimoto T, Shimojo N, et al. Physician-pharmacist cooperation program for blood pressure control in patients with hypertension: a randomized-controlled trial. Am J Hypertens. 2010;23: 1144-1152.
Integrated Pharmacy Research and Practice

\section{Publish your work in this journal}

Integrated Pharmacy Research and Practice is an international, peer-reviewed, open access, online journal, publishing original research, reports, reviews and commentaries on all areas of academic and professional pharmacy practice. This journal aims to represent the academic output of pharmacists and pharmacy practice with particular focus on integrated care. All papers are carefully peer reviewed

\section{Dovepress}

to ensure the highest standards as well as ensuring that we are informing and stimulating pharmaceutical professionals. The manuscript management system is completely online and includes a very quick and fair peer-review system, which is all easy to use. Visit http://www.dovepress.com/testimonials.php to read real quotes from published authors. 